\title{
Article \\ Comparison of Psoriatic Arthritis and Rheumatoid Arthritis Patients across Body Mass Index Categories in Switzerland
}

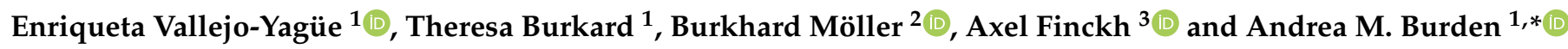 \\ 1 Department of Chemistry and Applied Biosciences, Institute of Pharmaceutical Sciences, ETH Zurich, \\ CH-8093 Zurich, Switzerland; enriqueta.vallejo@pharma.ethz.ch (E.V.-Y.); \\ theresa.burkard@pharma.ethz.ch (T.B.) \\ 2 Rheumatology, Immunology and Allergy, Inselspital, University Hospital of Bern, CH-3010 Bern, Switzerland; \\ burkhard.moeller@insel.ch \\ 3 Division of Rheumatology, University Hospitals of Geneva, CH-1206 Geneva, Switzerland; \\ axel.finckh@hcuge.ch \\ * Correspondence: andrea.burden@pharma.ethz.ch; Tel.: +41-76-685-22-56
}

check for updates

Citation: Vallejo-Yagüe, E.; Burkard, T.; Möller, B.; Finckh, A.; Burden, A.M. Comparison of Psoriatic Arthritis and Rheumatoid Arthritis Patients across Body Mass Index Categories in Switzerland. J. Clin. Med. 2021, 10, 3194. https:// doi.org/10.3390/jcm10143194

Academic Editors: Emmanuel Andres and Salvatore D'Angelo

Received: 14 May 2021

Accepted: 15 July 2021

Published: 20 July 2021

Publisher's Note: MDPI stays neutral with regard to jurisdictional claims in published maps and institutional affiliations.

Copyright: (c) 2021 by the authors. Licensee MDPI, Basel, Switzerland. This article is an open access article distributed under the terms and conditions of the Creative Commons Attribution (CC BY) license (https:// creativecommons.org/licenses/by/ $4.0 /)$

\begin{abstract}
Abnormal body mass index (BMI) was associated with worse rheumatic markers in psoriatic arthritis (PsA) and rheumatoid arthritis (RA). Aiming to describe PsA and RA patients stratified by BMI, we performed a descriptive study in PsA and RA patients (two distinct cohorts) in the Swiss Clinical Quality Management in Rheumatic Diseases (SCQM) registry. New users of biologic or targeted synthetic disease-modifying anti-rheumatic drugs (b/tsDMARDs) were stratified by BMI at the start of their treatment (underweight, normal weight, overweight, obese). The PsA underweight and normal weight categories were merged. Age at disease onset and further characteristics at the start of the first $\mathrm{b} / \mathrm{tsDMARD}$ treatment were compared across BMI categories vs. the corresponding normal weight group. The study included 819 PsA (36.5\% overweight, $23.8 \%$ obese) and 3217 RA patients (4.4\% underweight, $31.8 \%$ overweight, $17.0 \%$ obese). Compared to the corresponding normal weight group, PsA and RA obese patients had significantly $(p<0.05)$ higher $\mathrm{C}$-reactive protein, worse disease activity, and lower quality of life (QoL). Obese PsA patients had significantly worse skin manifestation and pain, while obese RA patients had significantly higher erythrocyte sedimentation rate and tender joint counts, as well as lower seropositive prevalence. To conclude, obese PsA and RA patients presented worse disease activity and poorer QoL than those with normal weight.
\end{abstract}

Keywords: psoriatic arthritis; rheumatoid arthritis; patient-reported outcomes; biologics

\section{Introduction}

Obesity represents an increasing healthcare burden worldwide [1]; it affects approximately $15 \%$ of the European population [2] and 11\% of the Swiss population [3]. Understanding obesity as a low-grade systemic inflammatory condition, where the white adipose tissue behaves as an endocrine organ secreting adipokines responsible for immune and inflammatory processes $[4,5]$, suggests a common pathological pathway with immune-mediated inflammatory rheumatic diseases.

Concerns about obesity or high body mass index (BMI) hindering the management of psoriatic arthritis (PsA) and rheumatoid arthritis (RA) patients have emerged in the past decade [4,6-9]. A higher prevalence of obesity was observed in PsA and RA patients compared to the general population [10-13], with PsA patients having the highest observed prevalence among both diseases $[11,14]$. Obesity was associated with worse disease activity and disease management in both PsA and RA [12,15-21] and a detrimental response to anti-tumor necrosis factor alpha (anti-TNF) treatments [15,17,18]. Additionally, despite the association of weight loss with a better disease outcome in PsA patients [22,23], a low BMI (underweight) was associated with worse RA disease activity $[10,24]$. 
Epidemiological studies using real-world data and clinical studies in PsA and RA patients rarely stratify by BMI, body weight, or body fat distribution. In a systematic review that included randomized clinical trials (RCTs) and observational studies assessing the failure to respond to anti-TNFs in adults with PsA, RA, spondyloarthropathies (SpA), and immune-mediated inflammatory diseases, the authors reported that less than $10 \%$ of eligible RCTs stratified by BMI at baseline [18].

We believe that there is interest and room for contribution to the scientific knowledge with regard to patient differences across BMI categories. Thus, we aimed to investigate differences in patient characteristics across BMI strata in patients with PsA and RA at the start of their first biologic or targeted synthetic disease-modifying anti-rheumatic drug (b/tsDMARD) treatment.

\section{Materials and Methods}

\subsection{Study Design and Data Source}

We performed a descriptive study of PsA and RA patients who were new users of $\mathrm{b} / \mathrm{tsDMARDs}$ and were registered in the Swiss Clinical Quality Management in Rheumatic Diseases (SCQM) database. The SCQM registry [25], initiated in 1997, is a national longitudinal population-based cohort of rheumatic diseases in Switzerland that includes PsA and RA patients. Informed consent is obtained prior to enrolment and patients can withdraw their consent at any time. The data is generated during patient consultations and inserted by both the rheumatologist and the patient. The collected information includes physician- and patient-reported clinical endpoints (e.g., pain, skin manifestation, inflammatory markers), composite disease activity scores, health surveys, treatments, and comorbidities. Antirheumatic medication is recorded by the rheumatologist, including information on the start and stop dates of each treatment regimen.

The study was reviewed by the ethics commission of the Canton of Zurich (KEK: Req-2020-00045). Since the researchers received pseudonymized data without access to the code key, a full ethics authorization was waived by the commission.

\subsection{Study Population}

PsA and RA patients registered in the SCQM database from 1 January 1997 to 31 July 2019 and starting their first $\mathrm{b} / \mathrm{tsDMARD}$ treatment were included in the study. The first recorded start date of $a b / t s D M A R D$ treatment in the SCQM was defined as the index date. We excluded patients that started their $b$ /tsDMARD treatment before the first registered visit in SCQM, as well as those without a measurement for weight and height at the index date (or within the 6 months prior to the index date). PsA and RA patients were treated as two distinct cohorts, which were analyzed separately but following a similar approach.

\subsection{Exposure}

The primary exposure of interest in this analysis was patient BMI strata at the start of their first $\mathrm{b} / \mathrm{tsDMARD}$ treatment. BMI $\left(\mathrm{kg} / \mathrm{m}^{2}\right)$ was calculated using the weight and height recorded at the index date, or as close as possible to this date within the previous 6 months (Supplementary Material Equation (S1)). We stratified patients by their BMI according to the World Health Organization (WHO) classification as follows: underweight (BMI < 18.5), normal weight (BMI 18.5-24.9), overweight (BMI 25.0-29.9), and obese (BMI $\geq 30$ [ [26]. The exposures of interest were the abnormal BMI categories (underweight, overweight, and obese), and the normal weight group was set as the comparator group.

\subsection{Covariates}

In both the PsA and RA patient cohorts, we included variables regarding disease onset, as well as patient characteristics, comorbidities, and medication use at the index date. Information on disease onset included age at first symptoms and age at diagnosis. Patient characteristics at the start of the first $\mathrm{b} / \mathrm{tsDMARD}$ treatment (baseline characteristics) included demographics (e.g., sex, age, BMI, life habits), patient- and physician-reported 
clinical endpoints (e.g., pain, skin manifestation), composite disease activity scores, and health or quality of life (QoL) questionnaires. These variables were collected at the index date, or as close as possible to that date within a 6-month look-back window. Chronic comorbidities were included if they were ever reported in the patients' records before or on the index date. Treatment with conventional synthetic disease-modifying antirheumatic drugs (csDMARDs) and glucocorticoids were identified if present on the index date. Disease-specific clinical endpoints and composite disease activity scores differed slightly between the PsA and RA patient cohorts. In both PsA and RA, we included the 28-joint disease activity score (DAS28) using the erythrocyte sedimentation rate (ESR) and DAS28 using C-reactive protein (CRP). While in the PsA cohort, minimal disease activity (MDA), the disease activity index for PsA (DAPSA), and the clinical DAPSA (cDAPSA) (DAPSA without CRP [27]) were additionally reported. Relevant formulas for composite disease activity scores and MDA are shown in the Supplementary Material Equations (S2)-(S6).

\subsection{Data Analysis}

All analyses were performed in each disease cohort separately. The BMI distribution was assessed in each disease cohort, and the prevalence of overweight and obese patients was plotted alongside the prevalence in the general Swiss population according to the Federal Statistical Office in Switzerland [3], stratifying by sex.

Patients' age at disease onset and patient baseline characteristics were described in each disease cohort, stratifying by BMI category. Categorical variables were presented with counts ( $n$-number of exposed patients) and percentages, and continuous variables were described using mean and standard deviation (SD) or median and interquartile range (IQR). Abnormal BMI categories were compared to the corresponding normal weight group using a chi-squared test for categorical variables and $t$-test or Wilcoxon test for continuous variables. For these tests, missing values did not function as a grouping variable, they were dropped instead. Statistical significance was defined as $p<0.05$.

Subsequently, among patients with a second $b /$ tsDMARD treatment during the study period, we described the prescription patterns for the first and second $b / t$ DDMARD treatments, stratifying by BMI.

In a post hoc analysis, we assessed the duration of first $b / t s D M A R D$ treatment across $\mathrm{BMI}$ categories and the reasons for treatment discontinuation. In this analysis, only patients with an available treatment stop date (or a start date of a second and different $b / t s D M A R D)$ were included.

The analyses and figures were performed using $\mathrm{R}$ statistical software, R Foundation for Statistical Computing (Vienna, Austria) [28] version 3.5.2 (20 December 2018), except for Figure 1, which was plotted using GraphPad Prism version 9.0.2 for Windows, GraphPad Software (San Diego, CA USA) [29]. 


\section{Overweight and obese prevalence}

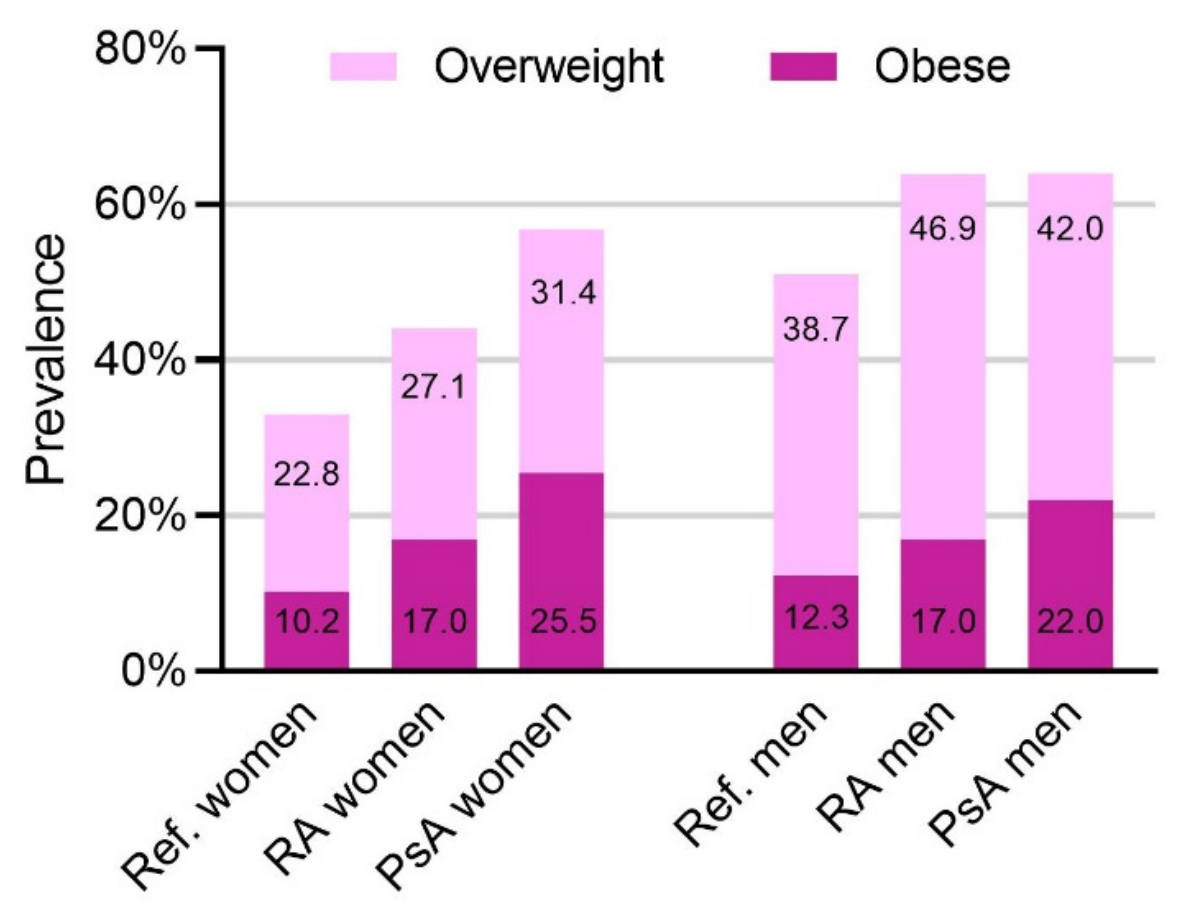

Figure 1. Overweight and obesity prevalence stratified by sex. The figure shows the findings from the studied rheumatoid arthritis (RA) and psoriatic arthritis (PsA) cohorts, along with the prevalence in the reference population (Ref.) according to the Swiss Federal Statistical Office, Übergewicht und Adipositas-Schweizerische Gesundheitsbefragung 2017-Korrigierte Version 25 September 2020 I Publikation. Bundesamt für Statistik 2020. Available at: https://www.bfs.admin.ch/bfs/ de/home/statistiken/gesundheit/erhebungen/sgb.assetdetail.14147705.html (accessed on 21 January 2021).

\section{Results}

We identified 4865 (1003 PsA and 3862 RA) patients in the SCQM between 1997 and 2019 who were new users of $a \mathrm{~b} / \mathrm{tsDMARD}$ and had available baseline information. From those, 829 patients were excluded due to missing weight and height information at the baseline. The remaining 819 PsA and 3217 RA patients were included in the study. A flow diagram reflecting the selection process is presented as Supplementary Figure S1.

The prevalence of overweight and obese patients in our study, compared to the Swiss national population and stratified by sex, is shown in Figure 1. For both PsA and RA, the prevalence of obesity and overweight was higher than in the general Swiss population for both males and females. For PsA, obesity prevalence was $25.47 \%$ in women and $22.03 \%$ in men, and in RA, it was $17.0 \%$ in both women and men.

\subsection{Patient Characteristics}

\subsubsection{Psoriatic Arthritis}

Among the 819 PsA patients, only 13 patients (1.59\%) had a BMI < 18.5; thus, due to the low numbers in the underweight category, we combined the underweight and normal weight groups in the PsA cohort. This resulted in 325 (39.68\%) normal weight, 299 (36.51\%) overweight, and 195 (23.81\%) obese PsA patients (Table 1). Compared to the normal weight group, PsA patients categorized as overweight and obese were significantly older at the age of first symptoms and diagnosis. 
Table 1. Age at disease onset in the psoriatic arthritis (PsA) patient cohort.

\begin{tabular}{|c|c|c|c|c|c|}
\hline & Normal Weight & Overweight & & Obese & \\
\hline & $(n=325)$ & $(n=299)$ & $p$ & $(n=195)$ & $p$ \\
\hline Age at first symptoms (mean (SD)) & $38.04(14.33)$ & $41.35(13.75)$ & 0.004 & $40.73(12.17)$ & 0.031 \\
\hline Missing & $7(2.15)$ & $8(2.68)$ & & $4(2.05)$ & \\
\hline Age at diagnosis (mean (SD)) & $41.81(14.39)$ & $45.21(13.09)$ & 0.002 & $45.00(11.58)$ & 0.010 \\
\hline Missing & $5(1.54)$ & $5(1.67)$ & & $5(2.56)$ & \\
\hline
\end{tabular}

Values are the number and column percentages, unless otherwise specified. The underweight, overweight, and obese categories were compared to the normal weight group using $t$-test. For the test, the missing values did not function as a grouping variable. Abbreviations: $n$-sample size; SD—standard deviation.

Approximately half of the patients were women, with the lowest frequency of women in the underweight group (44.48\%) and the highest in the normal weight category (56.31\%). The mean age in the overweight group (50.55 years (SD 12.57)) was significantly higher than in the normal weight group (47.42 (SD 13.59)).

In the PsA cohort, anti-TNF drugs were the first $\mathrm{b} / \mathrm{tsDMARDs}$ for $91.08 \%$ of normal weight, $91.64 \%$ of overweight, and $87.18 \%$ of obese patients (Table 2 ). Among those not treated with anti-TNFs, $68.97 \%$ normal weight, $64.00 \%$ overweight, and $68.00 \%$ obese patients received a tsDMARD (i.e., apremilast), and the remaining patients received a nonTNF biologic. History of cardiovascular event/disease and diabetes was more frequent in obese vs. the normal weight patients. Further information on the patients' characteristics is provided in Supplementary Table S1. Here we show that the frequency of higher education and physical activity decreased with increasing BMI categories, but the frequency of smoking and alcohol consumption was similar between the BMI groups.

Table 2. Demographics, medication, and comorbidities of the psoriatic arthritis (PsA) patients at the start of their first biologic or targeted synthetic disease-modifying anti-rheumatic drug (b/tsDMARD) treatment.

\begin{tabular}{|c|c|c|c|c|c|}
\hline & Normal Weight & Overweight & & Obese & \\
\hline & $(n=325)$ & $(n=299)$ & $p$ & $(n=195)$ & $p$ \\
\hline Women & $183(56.31)$ & $133(44.48)$ & 0.004 & $108(55.38)$ & 0.909 \\
\hline Age, years (mean (SD)) & $47.42(13.59)$ & $50.55(12.57)$ & 0.003 & $49.47(10.80)$ & 0.073 \\
\hline PsA duration, years (median [IQR]) & $2.47[0.60,7.05]$ & $2.67[0.66,7.58]$ & 0.969 & $1.79[0.68,5.80]$ & 0.290 \\
\hline Missing & $5(1.6)$ & $5(1.67)$ & & $5(2.56)$ & \\
\hline First $b /$ tsDMARD & & & 0.900 & & 0.369 \\
\hline Anti-TNF biologic & $296(91.08)$ & $274(91.64)$ & & $170(87.18)$ & \\
\hline Other biologic & $9(2.77)$ & $9(3.01)$ & & $8(4.1)$ & \\
\hline tsDMARD & $20(6.15)$ & $16(5.35)$ & & $17(8.72)$ & \\
\hline csDMARD on index date & $157(48.31)$ & $153(51.17)$ & 0.526 & $105(53.85)$ & 0.258 \\
\hline Glucocorticoids on index date & $40(12.31)$ & $38(12.71)$ & 0.976 & $18(9.23)$ & 0.350 \\
\hline Other rheumatological disease & $32(9.85)$ & 37 (12.37) & 0.380 & $26(13.33)$ & 0.281 \\
\hline Fractures, surgeries & $6(1.85)$ & $2(0.67)$ & 0.342 & $1(0.51)$ & 0.377 \\
\hline Skin problems, allergies, drug reactions & $36(11.08)$ & $47(15.72)$ & 0.112 & $25(12.82)$ & 0.647 \\
\hline Infections & $4(1.23)$ & $7(2.34)$ & 0.454 & $2(1.03)$ & 1.000 \\
\hline Cancerous tumor & $7(2.15)$ & $5(1.67)$ & 0.884 & $7(3.59)$ & 0.484 \\
\hline Cardiovascular event/disease & $27(8.31)$ & $40(13.38)$ & 0.056 & $33(16.92)$ & 0.005 \\
\hline Diabetes & $4(1.23)$ & $8(2.68)$ & 0.307 & $10(5.13)$ & 0.017 \\
\hline
\end{tabular}


Table 2. Cont.

\begin{tabular}{|c|c|c|c|c|c|}
\hline & Normal Weight & Overweight & & Obese & \\
\hline & $(n=325)$ & $(n=299)$ & $p$ & $(n=195)$ & $p$ \\
\hline Other metabolic problems & $6(1.85)$ & $13(4.35)$ & 0.113 & $7(3.59)$ & 0.346 \\
\hline Depression/anxiety & $13(4)$ & $17(5.69)$ & 0.426 & $10(5.13)$ & 0.700 \\
\hline
\end{tabular}

Values are the number and column percentages, unless otherwise specified. Significance tests compared the overweight and obese categories to the normal weight group using chi-squared test for categorical variables and $t$-test for continuous variables, except for the Wilcoxon test for the PsA duration. For these tests, the missing values did not function as a grouping variable. Abbreviations: $n-$ sample size; SD—standard deviation; IQR—interquartile range; anti-TNF—anti-tumor necrosis factor; tsDMARD—targeted synthetic disease-modifying anti-rheumatic drug; csDMARD—conventional synthetic disease-modifying anti-rheumatic drug. Note: csDMARD and glucocorticoids indicate use on the index date, and not as ever having used them before.

Clinical characteristics of PsA patients at the start of the first $b / t s D M A R D$ treatment are presented in Table 3. Obese patients had significantly higher CRP than the normal weight category $(p=0.020)$, and no significant differences were observed in the rheumatoid factor (RF) and human leukocyte antigen B27 (HLA-B27) between the BMI strata. While the physician's global disease activity assessment was similar between BMI strata, the physician's global skin manifestation was significantly worse in the overweight and obese vs. the normal weight group $(p<0.02)$. Similarly, compared to the normal weight group, patient-reported disease activity (0 to 10$)$ was significantly worse in the obese group, and both overweight and obese patients reported significantly worse joint pain (0 to 10). Conversely, the mean number of tender joint counts (TJC) was similar among BMI categories. Furthermore, the mean number of swollen joint counts (SJC) was significantly higher in the overweight vs. normal weight patients, but this was not consistent in the obese group. Additionally, no differences were observed across BMI groups regarding additional clinical manifestations (i.e., dactylitis, enthesitis, sacroilitis, spinal involvement, coxitis, peripheral arthritis, and nail manifestation).

Table 3. Clinical characteristics of psoriatic arthritis (PsA) patients at the start of their first biologic or targeted synthetic disease-modifying anti-rheumatic drug (b/tsDMARD) treatment.

\begin{tabular}{|c|c|c|c|c|c|}
\hline & Normal Weight & Overweight & & Obese & \\
\hline & $(n=325)$ & $(n=299)$ & $p$ & $(n=195)$ & $p$ \\
\hline RF+ & $14(4.31)$ & $10(3.34)$ & 0.667 & $5(2.56)$ & 0.423 \\
\hline Missing & $88(27.08)$ & $80(26.76)$ & & $52(26.67)$ & \\
\hline HLA-B27+ & $40(12.31)$ & $28(9.36)$ & 0.319 & $22(11.28)$ & 1.000 \\
\hline Missing & $150(46.15)$ & $142(47.49)$ & & $98(50.26)$ & \\
\hline ESR mm/h (median [IQR]) & $10(5,21.75)$ & $12(6 ., 22.25)$ & 0.104 & $14.5(6,23)$ & 0.081 \\
\hline Missing & $31(9.54)$ & $27(9.03)$ & & $13(6.67)$ & \\
\hline CRP mg/dL (median [IQR]) & $0.5(0.2,0.9)$ & $0.60(0.3,1.10)$ & 0.152 & $0.79(0.40,1.20)$ & 0.020 \\
\hline Missing & $40(12.31)$ & $36(12.04)$ & & $18(9.23)$ & \\
\hline $\begin{array}{l}\text { Physician global disease activity (mean } \\
\text { (SD)) }\end{array}$ & $4.43(2.03)$ & $4.56(1.86)$ & 0.414 & $4.43(1.85)$ & 0.991 \\
\hline Missing & $17(5.23)$ & $9(3.01)$ & & $6(3.08)$ & \\
\hline Physician global skin manifestation & $0.93(0.85)$ & $1.11(0.84)$ & 0.012 & $1.12(0.85)$ & 0.019 \\
\hline Missing & $30(9.23)$ & $17(5.69)$ & & $14(7.18)$ & \\
\hline $\begin{array}{l}\text { Patient-reported disease activity }(0-10) \\
\text { (mean (SD)) }\end{array}$ & $5.13(2.72)$ & $5.46(2.54)$ & 0.178 & $5.97(2.60)$ & 0.003 \\
\hline Missing & $89(27.38)$ & $59(19.73)$ & & $48(24.62)$ & \\
\hline Joint pain last $24 \mathrm{~h}(0-10)$ (mean (SD)) & $4.90(2.65)$ & $5.41(2.39)$ & 0.028 & $6.11(2.41)$ & $<0.001$ \\
\hline
\end{tabular}


Table 3. Cont.

\begin{tabular}{|c|c|c|c|c|c|}
\hline & Normal Weight & Overweight & & Obese & \\
\hline & $(n=325)$ & $(n=299)$ & $p$ & $(n=195)$ & $p$ \\
\hline Missing & $82(25.23)$ & $56(18.73)$ & & $46(23.59)$ & \\
\hline $\begin{array}{l}\text { Number tender joints } 28(0-28) \text { (mean } \\
\text { (SD)) }\end{array}$ & $4.21(5.11)$ & $4.90(5.52)$ & 0.109 & $4.35(5.10)$ & 0.767 \\
\hline Missing & $12(3.69)$ & $6(2.01)$ & & $10(5.13)$ & \\
\hline $\begin{array}{l}\text { Number tender joints } 68(0-68) \text { (mean } \\
(\mathrm{SD}) \text { ) }\end{array}$ & $8.07(9.08)$ & $8.97(10.23)$ & 0.267 & $8.65(9.63)$ & 0.518 \\
\hline Missing & $41(12.62)$ & $20(6.69)$ & & $21(10.77)$ & \\
\hline $\begin{array}{l}\text { Number swollen joints } 28 \text { (0-28) (mean } \\
\text { (SD)) }\end{array}$ & $2.73(3.39)$ & $3.65(4.57)$ & 0.005 & $2.88(3.36)$ & 0.627 \\
\hline Missing & $8(2.46)$ & $6(2.01)$ & & $8(4.1)$ & \\
\hline $\begin{array}{l}\text { Number swollen joints } 66(0-66) \text { (mean } \\
\text { (SD)) }\end{array}$ & $4.62(5.22)$ & $5.78(7.1)$ & 0.026 & $4.76(5.30)$ & 0.769 \\
\hline Missing & $41(12.62)$ & $21(7.02)$ & & $21(10.77)$ & \\
\hline Musculoskeletal manifestations & $269(82.77)$ & $242(80.94)$ & 0.624 & $163(83.59)$ & 0.904 \\
\hline Manifestation: dactylitis & $114(35.08)$ & $119(39.8)$ & 0.256 & $80(41.03)$ & 0.206 \\
\hline Manifestation: enthesitis & $138(42.46)$ & $120(40.13)$ & 0.611 & $81(41.54)$ & 0.909 \\
\hline Manifestation: sacroiliitis & $80(24.62)$ & $71(23.75)$ & 0.873 & $34(17.44)$ & 0.071 \\
\hline Manifestation: spinal involvement & $91(28)$ & $78(26.09)$ & 0.655 & $48(24.62)$ & 0.458 \\
\hline Manifestation: coxitis & $18(5.54)$ & $9(3.01)$ & 0.176 & $17(8.72)$ & 0.222 \\
\hline Manifestation: peripheral arthritis & $170(52.31)$ & $158(52.84)$ & 0.957 & $113(57.95)$ & 0.246 \\
\hline Nail manifestation & $68(20.92)$ & $71(23.75)$ & 0.453 & $56(28.72)$ & 0.056 \\
\hline
\end{tabular}

Values are the number and column percentages, unless otherwise specified. Significance tests compared the overweight and obese categories to the normal weight group using chi-squared test for categorical variables and $t$-test for continuous variables, except for the Wilcoxon test for ESR and CRP. For the tests, the missing values did not function as a grouping variable. Abbreviations: $n$-sample size; SD—standard deviation; IQR -interquartile range; RF+—rheumatoid factor positive; HLA-B27+—human leukocyte antigen B27 positive; ESR—erythrocyte sedimentation rate; $\mathrm{mm} / \mathrm{h}$ - millimeters per hour; $\mathrm{CRP}$-C-reactive protein; $\mathrm{mg} / \mathrm{dL}$-milligrams per deciliter.

The composite disease activity scores and health or QoL surveys of the PsA patients at the start of the first $b / t s D M A R D$ treatment are presented in Table 4. Overweight and obese patients presented worse disease activity, with significantly higher DAPSA (overweight 27.03 (SD 17.81), $p=0.022$; obese 26.90 (SD 15.33), $p=0.037$ ) compared to the normal weight group (23.23 (SD 15.46)). This was in line with the cDAPSA results and, likewise, significantly fewer patients had MDA in the overweight $(1.67 \%, p=0.002)$ and obese $(2.05 \%$, $p=0.026)$ vs. normal weight patients $(6.77 \%)$. However, DAS28 was only significantly higher in the overweight group. Regarding body function and QoL, obese patients, in comparison to the normal weight group, had consistently significantly worse measures on the following surveys: Health Assessment Questionnaire (HAQ), European Quality of Life-5 dimensions (EuroQoL EQ-5D), Dermatology Life Quality Index (DLQI), and Short Form containing 12 items (SF12) physical component summary (pcs). However, this was not observed in the SF12 mental component summary (mcs). 
Table 4. Composite disease activity scores and health or quality of life surveys in psoriatic arthritis (PsA) patients at the start of their first biologic or targeted synthetic disease-modifying anti-rheumatic drug (b/tsDMARD) treatment.

\begin{tabular}{|c|c|c|c|c|c|}
\hline & Normal Weight & Overweight & & Obese & \\
\hline & $(n=325)$ & $(n=299)$ & $p$ & $(n=195)$ & $p$ \\
\hline MDA & $22(6.77)$ & $5(1.67)$ & 0.002 & $4(2.05)$ & 0.026 \\
\hline Missing & $64(19.69)$ & $41(13.71)$ & & $36(18.46)$ & \\
\hline DAPSA (mean (SD)) & $23.23(15.46)$ & $27.03(17.81)$ & 0.022 & $26.90(15.33)$ & 0.037 \\
\hline Missing & $118(36.31)$ & $95(31.77)$ & & $72(36.92)$ & \\
\hline cDAPSA without CRP (mean (SD)) & $22.16(14.95)$ & $25.64(17.21)$ & 0.023 & $26.03(14.89)$ & 0.020 \\
\hline Missing & $106(32.62)$ & $72(24.08)$ & & $65(33.33)$ & \\
\hline DAS28-ESR (mean (SD)) & $3.30(1.27)$ & $3.57(1.32)$ & 0.014 & $3.43(1.23)$ & 0.273 \\
\hline Missing & $44(13.54)$ & $34(11.37)$ & & $24(12.31)$ & \\
\hline DAS28-CRP (mean (SD)) & $3.26(1.12)$ & $3.52(1.18)$ & 0.011 & $3.41(1.09)$ & 0.191 \\
\hline Missing & $51(15.69)$ & $42(14.05)$ & & $24(12.31)$ & \\
\hline HAQ (mean (SD)) & $0.71(0.65)$ & $0.75(0.58)$ & 0.375 & $0.89(0.61)$ & 0.003 \\
\hline Missing & $53(16.31)$ & $46(15.38)$ & & 38 (19.49) & \\
\hline Euro-QoL (mean (SD)) & $65.32(17.81)$ & $63.51(17.38)$ & 0.366 & $60.33(20.31)$ & 0.037 \\
\hline Missing & $169(52.00)$ & 145 (48.49) & & $90(46.15)$ & \\
\hline DLQI (mean (SD)) & $3.53(5.35)$ & $4.62(6.08)$ & 0.087 & $5.52(7.66)$ & 0.013 \\
\hline Missing & $167(51.38)$ & $137(45.82)$ & & $87(44.62)$ & \\
\hline SF12-pcs (mean (SD)) & $39.06(10.54)$ & $38.23(9.94)$ & 0.368 & $35.78(9.23)$ & 0.001 \\
\hline Missing & $67(20.62)$ & $64(21.4)$ & & $41(21.03)$ & \\
\hline SF12-mcs (mean (SD)) & $45.96(11.36)$ & $45.48(11.46)$ & 0.640 & $44.12(11.67)$ & 0.116 \\
\hline Missing & $67(20.62)$ & $64(21.4)$ & & $41(21.03)$ & \\
\hline
\end{tabular}

Values are the number and column percentages, unless otherwise specified. Significance tests compared the overweight and obese categories to the normal weight group using chi-squared test for categorical variables and $t$-test for continuous variables. For the tests, the missing values did not function as a grouping variable. Abbreviations: $n$-sample size; SD—standard deviation; MDA—minimal disease activity; DAPSA—Disease Activity Index for Psoriatic Arthritis; DAS28-28-joint Disease Activity Score; ESR—erythrocyte sedimentation rate; CRP-C-reactive protein; HAQ—Health Assessment Questionnaire; Euro-QoL_European Quality of Life instrument; DLQI-Dermatology Life Quality Index; SF12-Short-Form 12 health survey; pcs—physical component summary; mcs—mental component summary.

\subsubsection{Rheumatoid Arthritis}

Among the 3217 RA patients, 142 (4.41\%), 1505 (46.78\%), 1024 (31.83\%), and 546 $(16.97 \%)$ patients were classified as underweight, normal weight, overweight, and obese, respectively. The age of the RA patients at disease onset is provided in Table 5 . The patients categorized as overweight and obese were significantly older than the normal weight group at the age of first symptoms and diagnosis, and the underweight group was significantly younger at both dates.

Table 5. Age at disease onset in the rheumatoid arthritis (RA) patient cohort.

\begin{tabular}{|c|c|c|c|c|c|c|c|}
\hline & Normal Weight & Overweight & & Obese & & Underwei & \\
\hline & $(n=1505)$ & $(n=1024)$ & $p$ & $(n=546)$ & $p$ & $(n=142)$ & $p$ \\
\hline $\begin{array}{l}\text { Age at first symptoms, years (mean } \\
\text { (SD)) } \\
\text { Missing }\end{array}$ & $\begin{array}{c}43.90(15.46) \\
34(2.26)\end{array}$ & $\begin{array}{c}48.42(13.98) \\
39(3.81)\end{array}$ & $<0.001$ & $\begin{array}{c}48.52 \\
(12.59) \\
21(3.85)\end{array}$ & $<0.001$ & $\begin{array}{l}37.78 \\
(16.68) \\
7(4.93)\end{array}$ & $<0.001$ \\
\hline $\begin{array}{l}\text { Age at diagnosis, years (mean (SD)) } \\
\text { Missing }\end{array}$ & $\begin{array}{l}45.36(15.31) \\
\quad 34(2.26)\end{array}$ & $\begin{array}{c}50.10(13.71) \\
33(3.22)\end{array}$ & $<0.001$ & $\begin{array}{c}49.95 \\
(12.67) \\
19(3.48)\end{array}$ & $<0.001$ & $\begin{array}{l}39.86 \\
(16.87) \\
8(5.63)\end{array}$ & $<0.001$ \\
\hline
\end{tabular}

Values are the number and column percentages, unless otherwise specified. The underweight, overweight, and obese categories were compared to the normal weight group using $t$-test. For the test, the missing values did not function as a grouping variable. Abbreviations: $n$-sample size; SD—standard deviation. 
treatment, both overweight (mean 57.63 years (SD 12.28), $p<0.001$ ) and obese patients (mean 57.04 years (SD 11.65), $p<0.001$ ) were significantly older than the normal weight group (53.86 (SD 14.60)), and the underweight group was significantly younger (49.37 (SD 16.47), $p=0.001$ ). The first $\mathrm{b} / \mathrm{tsDMARD}$ was an anti-TNF in $89.44 \%$ of underweight, $87.51 \%$ of normal weight, $85.06 \%$ of overweight, and $84.07 \%$ of obese patients. Moreover, among those starting with another $\mathrm{b} / \mathrm{tsDMARD}, 20.00 \%$ of underweight, $22.34 \%$ of normal weight, $24.18 \%$ of overweight, and $18.39 \%$ of obese patients received a tsDMARD, while the remaining patients received a non-TNF biologic. Prior history of cardiovascular event/disease, diabetes, other rheumatologic diseases, and depression were significantly more frequent in overweight and obese patients, and fractures were less frequent in obese patients. Other complementary information on patient characteristics is provided in Supplementary Table S2.

Table 6. Demographics, medication, and comorbidities of the rheumatoid arthritis (RA) patients at the start of their first biologic or targeted synthetic disease-modifying anti-rheumatic drug (b/tsDMARD) treatment.

\begin{tabular}{|c|c|c|c|c|c|c|c|}
\hline & Normal Weight & Overweight & & Obese & & Underweight & \\
\hline & $(n=1505)$ & $(n=1024)$ & $p$ & $(n=546)$ & $p$ & $(n=142)$ & $p$ \\
\hline Women & $1237(82.19)$ & $664(64.84)$ & $<0.001$ & $416(76.19)$ & 0.003 & $133(93.66)$ & 0.001 \\
\hline Age, years (mean (SD)) & $53.86(14.60)$ & $57.63(12.28)$ & $<0.001$ & $57.04(11.65)$ & $<0.001$ & $49.37(16.47)$ & 0.001 \\
\hline $\begin{array}{l}\text { RA duration, years } \\
\text { (median [IQR]) } \\
\text { Missing }\end{array}$ & $\begin{array}{c}4.82(1.67,12.32) \\
34(2.26)\end{array}$ & $\begin{array}{c}4.08(1.35 \\
10.75) \\
33(3.22)\end{array}$ & 0.005 & $\begin{array}{c}3.73(1.38 \\
8.77) \\
19(3.48) \\
\end{array}$ & 0.001 & $\begin{array}{c}6.94(2.16 \\
14.47) \\
8(5.63) \\
\end{array}$ & 0.115 \\
\hline $\begin{array}{l}\text { First b/tsDMARD } \\
\text { Anti-TNF biologic } \\
\text { Other biologic } \\
\text { tsDMARD }\end{array}$ & $\begin{array}{c}1317(87.51) \\
146(9.7) \\
42(2.79)\end{array}$ & $\begin{array}{c}871(85.06) \\
116(11.33) \\
37(3.61)\end{array}$ & 0.192 & $\begin{array}{l}459(84.07) \\
71(13.00) \\
16(2.93)\end{array}$ & 0.095 & $\begin{array}{c}127(89.44) \\
12(8.45) \\
3(2.11)\end{array}$ & 0.785 \\
\hline csDMARD on index date & $1000(66.45)$ & $704(68.75)$ & 0.242 & $394(72.16)$ & 0.016 & $92(64.79)$ & 0.759 \\
\hline Glucocorticoids on index date & $604(40.13)$ & $427(41.7)$ & 0.456 & $222(40.66)$ & 0.870 & $49(34.51)$ & 0.222 \\
\hline Other rheumatological disease & $308(20.47)$ & $268(26.17)$ & 0.001 & $156(28.57)$ & $<0.001$ & $22(15.49)$ & 0.192 \\
\hline Fractures, surgeries & $151(10.03)$ & $80(7.81)$ & 0.067 & $36(6.59)$ & 0.021 & $14(9.86)$ & 1.000 \\
\hline Skin problems, allergies, drug reactions & $18(1.2)$ & $11(1.07)$ & 0.927 & $8(1.47)$ & 0.796 & $2(1.41)$ & 1.000 \\
\hline Infections & $22(1.46)$ & $17(1.66)$ & 0.816 & $12(2.2)$ & 0.338 & $1(0.7)$ & 0.718 \\
\hline Cancerous tumor & $27(1.79)$ & $26(2.54)$ & 0.253 & $12(2.2)$ & 0.683 & $1(0.7)$ & 0.535 \\
\hline Cardiovascular event/disease & $216(14.35)$ & $274(26.76)$ & $<0.001$ & $185(33.88)$ & $<0.001$ & $13(9.15)$ & 0.113 \\
\hline Diabetes & $36(2.39)$ & $49(4.79)$ & 0.002 & $48(8.79)$ & $<0.001$ & $2(1.41)$ & 0.650 \\
\hline Other metabolic problems & $36(2.39)$ & $55(5.37)$ & $<0.001$ & $33(6.04)$ & $<0.001$ & $0(0)$ & 0.118 \\
\hline Depression/anxiety & $58(3.85)$ & $58(5.66)$ & 0.041 & $41(7.51)$ & 0.001 & $5(3.52)$ & 1.000 \\
\hline
\end{tabular}

Values are the number and column percentages, unless otherwise specified. Significance tests compared the underweight, overweight, and obese categories to the normal weight group using chi-squared test for categorical variables and $t$-test for continuous variables, except for the Wilcoxon test for the RA duration. For the tests, the missing values did not function as a grouping variable. Abbreviations: $n$-sample size; SD—standard deviation; IQR - interquartile range; anti-TNF_-anti-tumor necrosis factor; tsDMARD—-targeted synthetic disease-modifying anti-rheumatic drug; csDMARD—conventional synthetic disease-modifying anti-rheumatic drug. Note: csDMARD and glucocorticoids indicate use on the index date, and not as ever having used them before.

Clinical characteristics, composite disease activity scores, and health or QoL surveys of RA patients at the start of their $b / t s D M A R D$ treatment are presented in Table 7. Significantly fewer RF+ patients were observed in the obese $(62.82 \%, p<0.001)$ vs. normal weight groups $(72.16 \%)$. Likewise, the prevalence of anti-CCP+ patients was significantly lower in obese $(43.59 \%, p<0.001)$ than in normal weight patients $(48.64 \%)$. Obese patients presented significantly higher ESR and CRP levels than the normal weight group. While the physician's global disease activity assessment remained similar across the RA BMI strata, the TJC were significantly increased in overweight and obese patients vs. the normal weight group, and this was not consistent with the SJC. 
Table 7. Clinical characteristics, composite disease activity scores, and health or quality of life surveys of rheumatoid arthritis (RA) patients at the start of their first biologic or targeted synthetic disease-modifying anti-rheumatic drug (b/tsDMARD) treatment.

\begin{tabular}{|c|c|c|c|c|c|c|c|}
\hline & Normal Weight & Overweight & & Obese & & Underweight & \\
\hline & $(n=1505)$ & $(n=1024)$ & $p$ & $(n=546)$ & $p$ & $(n=142)$ & $p$ \\
\hline $\begin{array}{l}\mathrm{RF}+ \\
\text { Missing }\end{array}$ & $\begin{array}{c}1086(72.16) \\
53(3.52)\end{array}$ & $\begin{array}{l}700(68.36) \\
58(5.66)\end{array}$ & 0.219 & $\begin{array}{c}343(62.82) \\
28(5.13)\end{array}$ & $<0.001$ & $\begin{array}{l}101(71.13) \\
4(2.82)\end{array}$ & 0.755 \\
\hline $\begin{array}{l}\text { Anti-CCP+ } \\
\text { Missing }\end{array}$ & $\begin{array}{l}732(48.64) \\
427(28.37)\end{array}$ & $\begin{array}{l}489(47.75) \\
278(27.15)\end{array}$ & 0.317 & $\begin{array}{l}238(43.59) \\
136(24.91)\end{array}$ & $<0.001$ & $\begin{array}{l}61(42.96) \\
49(34.51)\end{array}$ & 0.732 \\
\hline $\begin{array}{l}\text { ESR mm/h (median [IQR]) } \\
\text { Missing }\end{array}$ & $\begin{array}{l}18(9,32) \\
72(4.78)\end{array}$ & $\begin{array}{c}20(10,34) \\
37(3.61)\end{array}$ & 0.103 & $\begin{array}{l}20(10,33) \\
27(4.95)\end{array}$ & 0.026 & $\begin{array}{c}18(8,38.75) \\
4(2.82)\end{array}$ & 0.919 \\
\hline $\begin{array}{l}\text { CRP mg/dL (median [IQR]) } \\
\text { Missing }\end{array}$ & $\begin{array}{l}0.8(0.30,1.4) \\
851(56.54)\end{array}$ & $\begin{array}{l}0.8(0.3,1.6) \\
489(47.75)\end{array}$ & 0.089 & $\begin{array}{l}0.9(0.4,1.52) \\
251(45.97)\end{array}$ & 0.005 & $\begin{array}{l}0.8(0.2,1.50) \\
97(68.31)\end{array}$ & 0.723 \\
\hline $\begin{array}{l}\text { Physician global disease } \\
\text { activity }(0-10) \text { (mean (SD)) } \\
\text { Missing (\%) }\end{array}$ & $\begin{array}{l}4.88(2.14) \\
534(35.48)\end{array}$ & $\begin{array}{l}4.87(2.14) \\
339(33.11)\end{array}$ & 0.926 & $\begin{array}{l}4.87(1.99) \\
178(32.6)\end{array}$ & 0.962 & $\begin{array}{l}5.02(2.13) \\
55(38.73)\end{array}$ & 0.547 \\
\hline $\begin{array}{l}\text { Number of tender joints } 28(0-28)(\text { mean }(S D)) \\
\text { Missing }(\%)\end{array}$ & $\begin{array}{c}6.31(6.32) \\
10(0.66)\end{array}$ & $\begin{array}{l}7.20(6.97) \\
6(0.59)\end{array}$ & 0.001 & $\begin{array}{l}7.19(6.7) \\
3(0.55)\end{array}$ & 0.007 & $\begin{array}{l}5.69(6.15) \\
2(1.41)\end{array}$ & 0.260 \\
\hline $\begin{array}{l}\text { Number of swollen joints } 28(0-28) \text { (mean (SD)) } \\
\text { Missing }(\%)\end{array}$ & $\begin{array}{l}6.68(5.9) \\
7(0.47)\end{array}$ & $\begin{array}{l}6.71(5.63) \\
2(0.2)\end{array}$ & 0.893 & $\begin{array}{l}6.30(5.50) \\
2(0.37)\end{array}$ & 0.198 & $\begin{array}{l}7.45(6.66) \\
1(0.7)\end{array}$ & 0.139 \\
\hline $\begin{array}{l}\text { DAS28-ESR (mean (SD)) } \\
\text { Missing }\end{array}$ & $\begin{array}{l}4.23(1.42) \\
81(5.38)\end{array}$ & $\begin{array}{l}4.39(1.41) \\
43(4.2)\end{array}$ & 0.007 & $\begin{array}{l}4.41(1.35) \\
29(5.31)\end{array}$ & 0.011 & $\begin{array}{l}4.22(1.57) \\
6(4.23)\end{array}$ & 0.946 \\
\hline $\begin{array}{l}\text { DAS28-CRP (mean (SD)) } \\
\text { Missing }\end{array}$ & $\begin{array}{l}3.92(1.20) \\
860(57.14)\end{array}$ & $\begin{array}{l}4.07(1.21) \\
495(48.34)\end{array}$ & 0.035 & $\begin{array}{l}4.12(1.12) \\
252(46.15)\end{array}$ & 0.016 & $\begin{array}{l}3.90(1.13) \\
98(69.01)\end{array}$ & 0.906 \\
\hline $\begin{array}{l}\text { HAQ (mean (SD)) } \\
\text { Missing }\end{array}$ & $\begin{array}{l}0.96(0.71) \\
122(8.11)\end{array}$ & $\begin{array}{l}1.07(0.72) \\
104(10.16)\end{array}$ & $<0.001$ & $\begin{array}{l}1.18(0.75) \\
71(13)\end{array}$ & $<0.001$ & $\begin{array}{l}1.06(0.74) \\
11(7.75)\end{array}$ & 0.125 \\
\hline $\begin{array}{l}\text { Euro-QoL (mean (SD)) } \\
\quad \text { Missing }\end{array}$ & $\begin{array}{l}65.85(19.31) \\
945(62.79)\end{array}$ & $\begin{array}{c}60.75(21.80) \\
587(57.32)\end{array}$ & $<0.001$ & $\begin{array}{c}59.02(22.79) \\
308(56.41)\end{array}$ & $<0.001$ & $\begin{array}{c}64.74(18.16) \\
105(73.94)\end{array}$ & 0.735 \\
\hline $\begin{array}{l}\text { SF12-pcs (mean (SD)) } \\
\text { Missing }\end{array}$ & $\begin{array}{l}36.47(10.27) \\
262(17.41)\end{array}$ & $\begin{array}{l}34.87(9.57) \\
208(20.31)\end{array}$ & $<0.001$ & $\begin{array}{l}34.16(9.79) \\
131(23.99)\end{array}$ & $<0.001$ & $\begin{array}{l}34.78(10.62) \\
24(16.9)\end{array}$ & 0.088 \\
\hline $\begin{array}{l}\text { SF12-mcs (mean (SD)) } \\
\text { Missing }\end{array}$ & $\begin{array}{l}46.01(11.56) \\
262(17.41)\end{array}$ & $\begin{array}{l}45.31(12.01) \\
208(20.31)\end{array}$ & 0.185 & $\begin{array}{c}44.80(12.33) \\
131(23.99)\end{array}$ & 0.070 & $\begin{array}{l}45.34(12.28) \\
24(16.9)\end{array}$ & 0.552 \\
\hline
\end{tabular}

Values are the number and column percentages, unless otherwise specified. Significance tests compared the underweight, overweight, and obese categories to the normal weight group using chi-squared test for categorical variables and $t$-test for continuous variables, except for the Wilcoxon test for ESR and CRP. For the tests, the missing values did not function as a grouping variable. Abbreviations: $n-$ sample size; $\mathrm{SD}$-standard deviation; IQR -interquartile range; RF+—rheumatoid factor positive; anti-CCP+—anti-cyclic citrullinated peptide positive; ESR — erythrocyte sedimentation rate; CRP—C-reactive protein; DAS28-28-joint Disease Activity Score; HAQ—Health Assessment Questionnaire; Euro-QoL-European Quality of Life instrument; SF12-Short-Form 12 health survey; pcs—physical component summary; mcs-mental component summary.

Regarding disease activity, DAS28-ESR was significantly higher in overweight (4.39 (SD 1.41), $p=0.007$ ) and obese (4.41 (SD 1.35), $p=0.011$ ) in comparison to normal weight patients (4.23 (SD 1.42)), and this finding was in agreement with the DAS28-CRP results. Additionally, compared to normal weight patients, the overweight and obese patients presented worse QoL according to the HAQ, Euro-QoL, and SF12-pcs, but not SF12-mcs. Slightly worse HAQ and SF12-pcs were observed in the underweight patients; however, this was not statistically significant.

\subsection{Treatment Trends for the First and Second b/tsDMARD Treatments}

\subsubsection{Psoriatic Arthritis}

In the PsA cohort, 385 patients $(47.00 \%)$ had a second $\mathrm{b} /$ tsDMARD following the stop of their first $b / t s D M A R D$ treatment. The distribution of paired first and second b/tsDMARDs used by these patients is illustrated in Figure 2, and counts to complement the figure are provided in the Supplementary Table S3. Among those with a recorded second b/tsDMARD treatment, $94.84 \%$ of normal weight, $95 \%$ of overweight, and $90 \%$ of obese patients were treated with an anti-TNF as their first $\mathrm{b} /$ tsDMARD. Among the patients starting with an anti-TNF treatment, $84.35 \%$ of normal weight, $85.71 \%$ of overweight, and $88.89 \%$ of obese patients moved to the same or another anti-TNF as their second treatment. The most common second $\mathrm{b} / \mathrm{tsDMARDs}$ across the four BMI categories continued being the anti-TNFs adalimumab, etanercept, golimumab, and infliximab. 


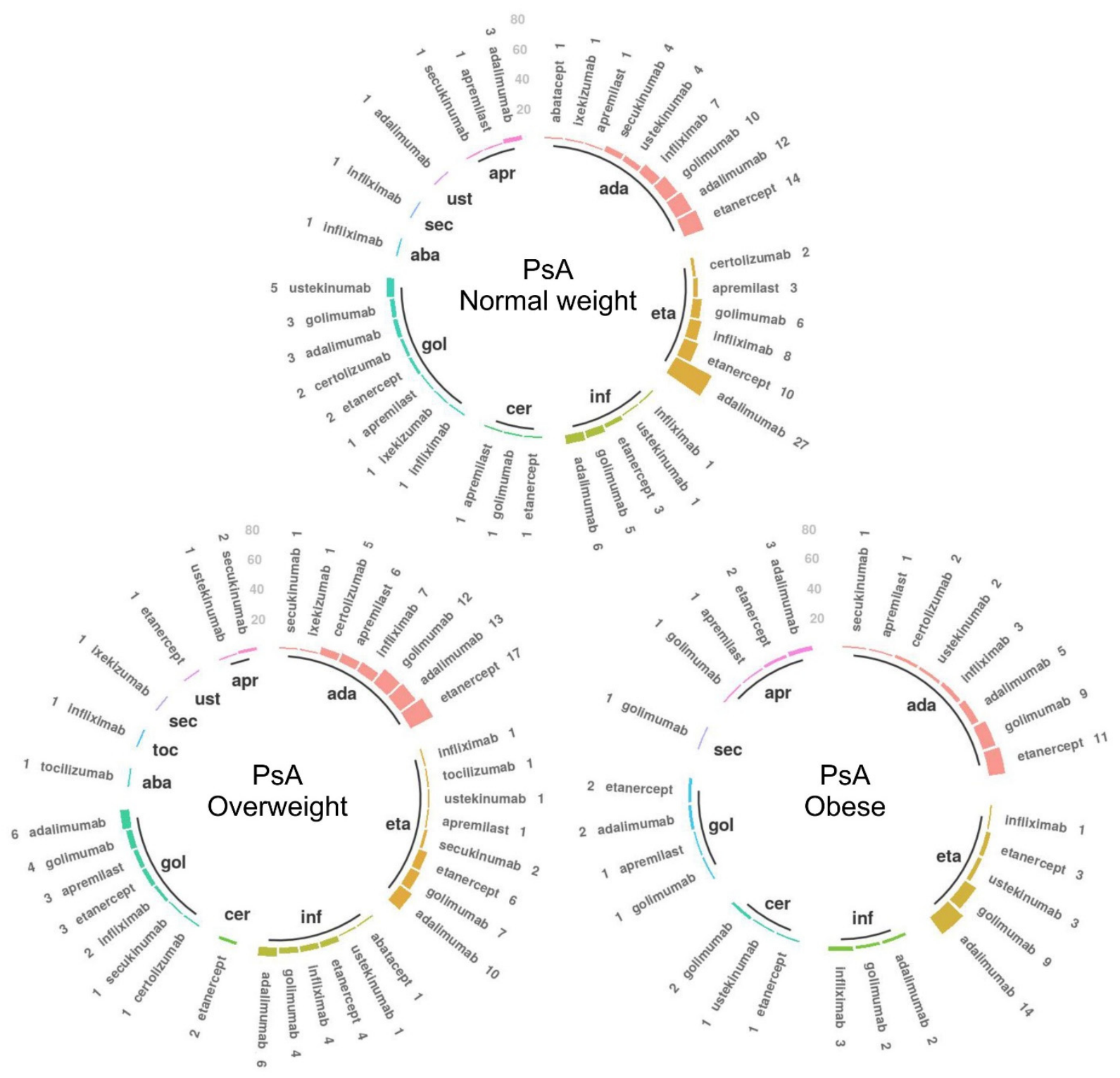

Figure 2. Treatment trends in the psoriatic arthritis (PsA) patients receiving a second biologic or targeted synthetic diseasemodifying anti-rheumatic drug $(\mathrm{b} / \mathrm{tsDMARD})$, stratified by body mass index $(n=385)$. The inner circle illustrates the first $b / t s D M A R D$, and for each corresponding initial drug, the bars indicate the second $b /$ tsDMARD treatment. Only patients with recorded second treatments are represented. The number of patients for each drug sequence is mentioned by each bar. Complementary data are provided in Supplementary Table S3. Abbreviations: PsA—psoriatic arthritis; ada— adalimumab; eta—etanercept; inf-infliximab; cer—certolizumab; gol—golimumab; aba—abatacept; toc—tocilizumab; sec-secukinumab; ust-ustekinumab; apr-apremilast.

In the post hoc analysis, we identified 451 patients (175 normal weight patients, 173 overweight, and 103 obese) with an available stopping date for their first $\mathrm{b} / \mathrm{tsDMARD}$ treatment. Among these, the median years of treatment were 11.66 (IQR $(3.91,24.28)$ ), 11.47 (IQR $(5.22,26.68))$, and 9.59 (IQR $(3.94,20.78)$ ) for normal weight, overweight, and obese PsA patients, respectively. No statistically significant differences were found in the duration of treatment across BMI groups. The reasons for stopping the first $b / t s D M A R D$ treatment (Supplementary Table S1) were statistically different in the obese $(p=0.024)$ versus normal weight strata. 


\subsubsection{Rheumatoid Arthritis}

In the RA cohort, 1546 patients $(48.06 \%$ ) received a second $\mathrm{b} / \mathrm{tsDMARD}$ following the stop of their first $\mathrm{b} / \mathrm{tsDMARD}$ treatment. Their distribution of paired first and second treatments are shown in Figure 3, complemented with numerical values in Supplementary Table S4. Following anti-TNFs as first treatment (as was the case in $96.61 \%$ of underweight patients, $91.99 \%$ of normal weight, $89.14 \%$ of overweight, $86.49 \%$ of obese), a total of $78.95 \%$ of underweight, $64.15 \%$ of normal weight, $61.83 \%$ of overweight, and $58.04 \%$ of obese patients continued with anti-TNF as a second $b /$ tsDMARD. Overall, the most commonly used treatments as a second $\mathrm{b} / \mathrm{tsDMARD}$ were adalimumab and etanercept, except for the obese group, where rituximab was more frequently used than etanercept.
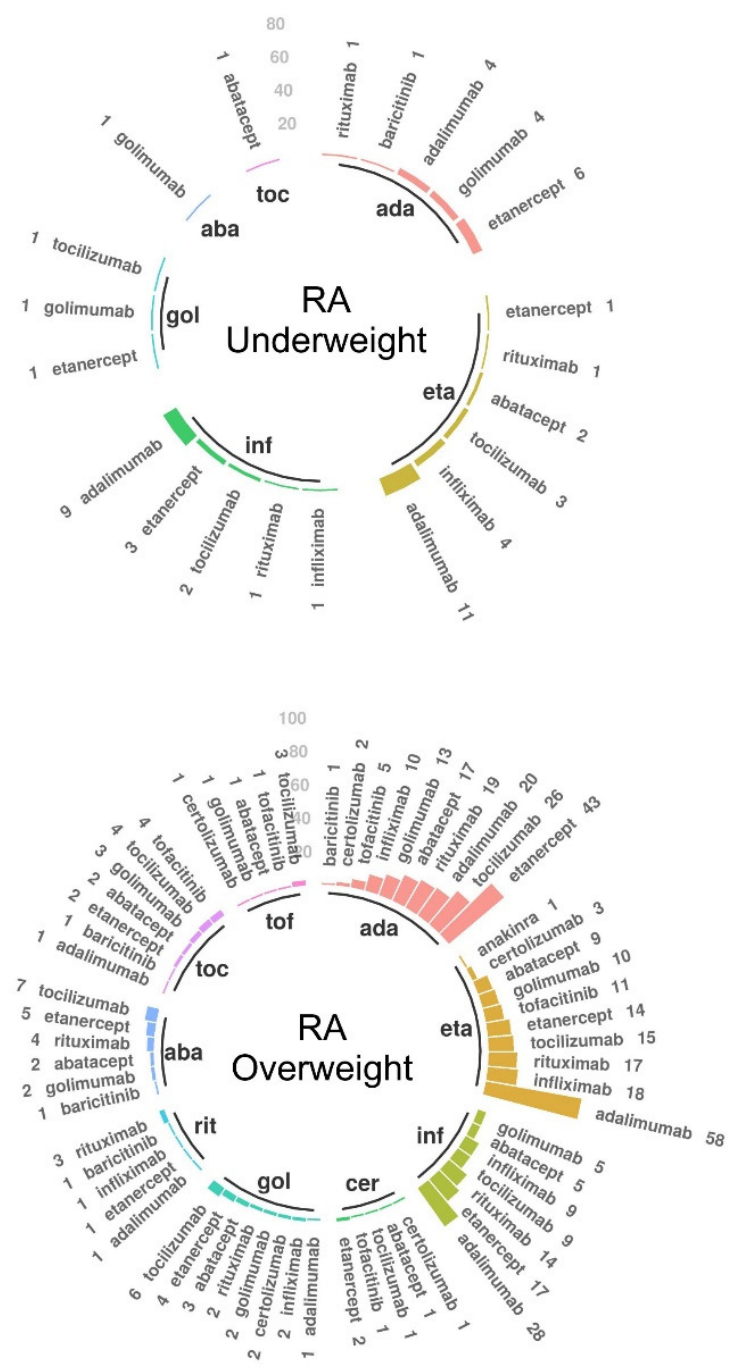
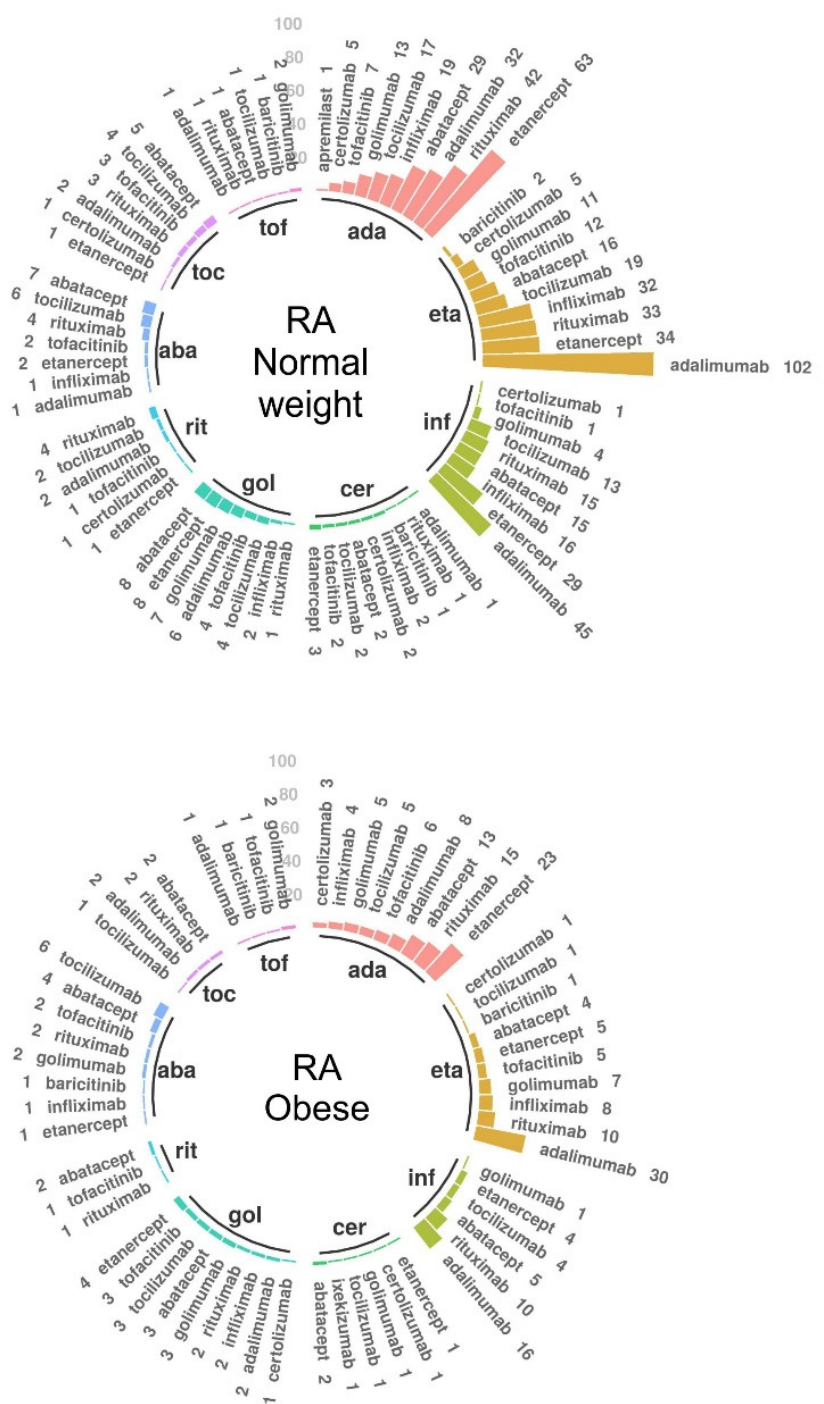

Figure 3. Treatment trends in the rheumatoid arthritis (RA) patients receiving a second biologic or targeted synthetic disease-modifying anti-rheumatic drug (b/tsDMARD), stratified by body mass index $(n=1546)$. The inner circle illustrates the first $b / t s D M A R D$, and for each corresponding initial drug, the bars indicate the second b/tsDMARD treatment. Only patients with recorded second treatments are represented. The number of patients for each drug sequence is mentioned by each bar. Complementary data are provided in Supplementary Table S4. Abbreviations: ada—adalimumab; eta—etanercept; inf-infliximab; cer—certolizumab; gol—golimumab; rit—rituximab; aba-abatacept; toc—tocilizumab; tof-tofacitinib.

The post hoc analysis identified 1787 patients (856 normal weight, 557 overweight, 307 obese, and 67 underweight) with available stop dates for their first b/tsDMARD treatment. Among these, the median years of treatment was 13.65 (IQR $(5.79,29.93)), 13.24$ (IQR (6.01, 
32.03)), 11.70 (IQR $(5.31,22.83))$, and 12.19 (IQR (5.14, 35.09)) years for patients with normal weight, overweight, obesity, and underweight, respectively. In comparison to the normal weight group, obese patients had a significantly shorter duration of treatment $(p=0.006)$. The distribution of reasons for stopping the first $b / t$ sDMARD treatment (Supplementary Table S2) was significantly different in obese vs. normal weight patients $(p=0.007)$.

\section{Discussion}

To our knowledge, this is one of the largest studies to examine differences in patient characteristics between PsA patients across BMI strata, and we additionally provided a comparison to the RA population. In our analysis, we identified that obese patients were generally older at disease onset and they had significantly higher CRP levels, worse disease activity scores, and lower QoL at the time of starting their first b/tsDMARD treatment compared to normal weight patients. Obese PsA patients also had worse skin manifestations and reported higher pain compared to the normal weight group. Meanwhile, in the RA cohort, the obese patients had higher ESR, higher TJC, but similar SJC, and smaller prevalence of RF+ patients. In both cohorts, anti-TNF drugs were the most commonly prescribed $\mathrm{b} / \mathrm{tsDMARDs}$ across every BMI category, and $>84 \%$ PsA and $>58 \%$ RA patients moved to the same or another anti-TNF (assuming gaps of $\geq 1$ month as treatment stoppages).

\subsection{Prevalence of BMI Strata}

We identified a higher obesity prevalence in our PsA (23.8\% obese) and RA (17.0\% obese) patient cohorts in comparison to the general Swiss population (Switzerland 2017, $11 \%$ obesity) [3]. This is in accordance with prior studies. For example, obesity prevalence was 32\% among PsA patients from Danish and Icelandic registries [15] compared to the $14-17 \%$ and $22 \%$ of the general Danish and Icelandic populations, respectively $[15,30,31]$. In Canadian studies, obesity prevalence was $35.4-37 \%$ in PsA $[12,13]$ and $28 \%$ in RA vs. $18 \%$ in the general population [11]. Furthermore, among RA patients in German cohorts, obesity prevalence was $21.4-23.8 \%$ vs. the $18.2 \%$ in the general population [10]. While we observed a lower prevalence of obesity in both our cohorts compared to the abovementioned studies, this may be explained by the less frequent obesity in our reference population. Additionally, our findings confirmed the higher obesity prevalence among PsA vs. RA patients, which were previously observed by other studies [11,14].

While we aimed to provide stratified information on underweight patients in both cohorts, we were unable to do so for the PsA cohort due to the small sample size. With 13 patients being underweight $(\mathrm{BMI}<18.5)$, the prevalence in our cohort was $1.59 \%$. Due to the lack of existing information on underweight PsA patients, it is unclear whether this is comparable across international patient cohorts. Conversely, in our RA cohort, $4.4 \%$ of patients were categorized as underweight. Comparing this prevalence with that in other studies, we observed a wide range of findings. In German cohorts, the prevalence of underweight patients was $1.1-2.2 \%$ in RA vs. $0.8 \%$ in the reference population [10]. In a Dutch cohort of active RA, 8 patients $(9.0 \%)$ had BMI $<20 \mathrm{~kg} / \mathrm{m}^{2}$ [32], and in a study in the United States, 38 patients $(4.9 \%)$ had BMI $<20 \mathrm{~kg} / \mathrm{m}^{2}$ [33]. While there seems to be a lack of agreement on the prevalence of underweight patients in RA, this may be explained by the use of different BMI thresholds and measuring time-points throughout the course of the disease, the potentially different underweight prevalence in the reference populations, and the restrictions posed by the study-specific inclusion/exclusion criteria. Further evidence on underweight patients in both PsA and RA are certainly warranted.

\subsection{Patient Characteristics}

Previous studies reported that overweight and obese PsA and RA patients were older than the normal weight patients at disease onset $[10,13]$, and this was similarly observed in our study. While the reasons for a later onset age are unclear, it could be the consequence of failing to recognize rheumatic clinical signs when they could be attributed to heavy body weight [13]. 
In an observational cohort study in Danish and Icelandic registries including 1271 PsA patients starting anti-TNFs, the authors reported that obese patients were older and had higher CRP levels, TJC, DAS28, HAQ, pain levels, and global disease assessments than nonobese patients at the baseline, but not significantly higher SJC [15]. Similarly, in our study, obese PsA patients had higher CRP levels, worse pain, and worse HAQ when compared to the normal weight group. However, SJC and DAS28 were only significantly higher in the overweight group, despite DAPSA showing significantly worse disease activity in both overweight and obese patients. This inconsistency between the PsA-specific DAPSA and the RA-derived DAS28 without specific adaptations to PsA in the obese group may be explained by the different components contributing to each score. First, DAPSA includes patient assessments of disease activity and pain, which were significantly higher in obese patients. Second, if the potential underestimation of SJC in obese patients due to excess fat tissue around the joints has a higher impact on the resulting DAS28 score than in DAPSA, this may also contribute to the significantly worse observed DAPSA score in both overweight and obese patients, but only significantly worse DAS28 in overweight compared to the normal weight patients.

Among the RA patients, some studies distinguished subjective (e.g., TJC, patient global assessment, and pain) and objective (e.g., CRP level, ESR, and SJC) endpoints when comparing obese and non-obese patients $[19,34]$. For example, in a systematic review and meta-analysis in which DAS28 and HAQ were higher in obese patients, the authors suggested that the increased disease activity was mainly due to elevated subjective score components, such as TJC, and global pain and health assessments [34]. Additionally, another systematic review and meta-analysis also suggested that obesity may influence patient global disease assessment, pain, and QoL, but agreed that SJC are not higher in obese RA patients [16]. Albrecht et al. similarly observed increased DAS28 but not SJC among obese RA patients in comparison to the normal weight ones, but conversely, with other studies, they also found higher ESR in obese patients [10]. In line with this, we identified worse DAS28, lower QoL, higher TJC, and no differences in SJC in obese compared to normal weight RA patients, but we also identified increased CRP levels and ESR in the obese vs. normal weight patients. Thus, we believe that the observed increased DAS28 in obese patients with RA was not only due to TJC, previously described as a subjective endpoint, but also due to the enhanced inflammatory markers (CRP level and ESR), or so-called objective end-points.

The discussion of objective and subjective endpoints is likely important for the assessment of obese rheumatology patients. While in the clinic, SJC is considered an objective measure, the excess or absence of fat tissue around the joints may influence the assessment of swelling, adding complexity to the practice and potentially reducing the reliability of this measure in obese patients. This may explain the inconsistency observed between SJC and TJC among RA patients, as well as the controversial higher SJC in overweight vs. obese among PsA patients. Additionally, in our RA cohort, we observed a tendency of higher SJC and lower TJC in underweight compared to normal weight patients, which contrasts the findings in obese patients. This SJC trend in underweight RA patients was also observed in German cohorts, along with heterogeneous results for TJC [10]. Following the above-discussed potential impact of fat mass in the SJC, we believe that higher SJC in underweight patients could be the consequence of a more easily detectable inflammation during clinical assessment and, conversely, SJC could potentially be underestimated in obese patients. Conversely, one may also consider that the disagreement between higher TJC, but similar SJC, in obese RA patients could be alternatively explained by hyperalgesia due to mood disorders, such as depression, and subsequent fibromyalgia, which has been associated with obesity [35].

Overall, obesity was associated with pain, lower QoL, higher disability, depression [36,37], cardiovascular risk [38], and diabetes [39] in the general population. Obesity (adiposity) was identified as a risk factor for PsA [40] and RA [41], and a higher frequency of lipid abnormalities (higher dyslipidemia and serum triglycerides and lower high-density 
lipoprotein (HDL)-cholesterol) was identified in PsA patients [42]. This, together with the evidence of white adipose tissue enhancing immune and inflammatory processes [4,5,43], and with the higher prevalence of obesity in PsA and RA patients vs. the general population [10-13], support the reason to believe that both the obesity and the rheumatology disease contribute to the patient status, and therefore may play a role in the assessed worse QoL and fragility observed in these patients. Additionally, the disagreement on the findings through QoL surveys, including physical components (SF12-pcs, Euro-QoL), vs. the SF12-mcs, which solely assesses mental components, suggests that the findings of worse QoL in PsA/RA obese patients may be driven by the physical restrictions and less so from potentially decreased mental wellbeing.

\subsection{Treatment Trends}

Existing studies have shown that obesity may be associated with a detrimental response to anti-TNF treatments in PsA and RA patients in comparison to non-obese or normal weight patients $[15,17,18]$. Conversely, it has been suggested that high BMI does not influence the response to abatacept [44-47], rituximab [48], and tocilizumab [49,50] in RA. Thus, while we observed that anti-TNFs were the most frequent choice of $b / t s D M A R D$ treatment across every BMI category in both cohorts, there was a slight tendency for lower anti-TNF use among RA patients in higher BMI categories, which could indicate that clinical decision-making is in line with these previous findings. However, this cannot be confirmed based on our results, and a time-series analysis may be more appropriate.

\subsection{Strengths and Limitations}

This is one of the largest studies that assessed differences in PsA patients across BMI categories and provides a further comparison with RA patients. To the best of our knowledge, the SCQM database is one of the few rheumatic disease registries with relatively complete information on patient weight and height, thereby enabling a stratification by BMI categories. Additionally, with obesity rates differing between countries [3,51], we consider the Swiss population a sample of interest due to its relatively low, although increasing, prevalence of obesity in the general population (2017, 11\%) [3]. However, BMI does not provide information on body composition [52], and therefore, we acknowledge the potential misclassification for those patients for whom the BMI categories do not fairly represent their fat distribution. Ideally, we would like to have data from the hip/waist circumference, the skinfold thickness, and the bioelectrical impedance, which would provide a better assessment of unhealthy weight. However, this was not available in our data. Moreover, this information is rarely recorded during clinical practice; thus, this is likely unrealistic to be present in real-world data. Missing information regarding body mass composition may be especially relevant for the underweight group, resulting in a lack of figures on cachexia (abnormal body composition). In cachexic patients, body fat (particularly belly fat) may remain stable or even increased despite muscle loss and weight loss [53]. Thus, it is possible that systemic inflammation in cachexic underweight patients may overlap with the rheumatic inflammation in a similar manner than in obese patients, despite the different phenotype. This could be better captured by more precise measures of body fat composition. However, we did not observe aggravated inflammatory markers in the underweight category vs. the normal weight patients, which may suggest the absence of additional systemic inflammation in these patients.

While the SCQM is a comprehensive dataset, we acknowledge that there are limitations regarding data completeness. We may have incomplete information on non-rheumatic comorbidities, as this information is self-reported by the patient and reporting systems have changed since the launch of the SCQM. For example, while previous evidence suggested that the prevalence of depression was 9-22\% in PsA [54-56] and 14-38\% in RA patients [57], we observed a lower prevalence (4-5.7\% in PsA and 3.5-7.5\% in RA), supporting the belief that comorbidities may be underreported in our cohorts. 
In both PsA and RA patients, we observed that obese patients were generally older than the normal weight patients, which could be due to their increasing BMI with age, and therefore the interplay between age, BMI, and disease activity in PsA and RA patients deserves further attention. Additionally, we stratified patients according to their BMI at the start of their first $b / t s D M A R D$ treatment; thus, for our analysis of differences in age at disease onset, we assumed that BMI remained constant from disease onset to the start of their $\mathrm{b} / \mathrm{tsDMARD}$ treatment.

Finally, the decision regarding what qualifies as a true treatment stop, or which length of treatment-free gap (between stop and restart of the same drug agent) should be considered as treatment continuation or as a true stop and restart may be arguable. In this study, we accepted a one-month grace period, whereby patients with a stop and restart of the same $b /$ tsDMARD agent were considered as having continuous use. Therefore, the observed high proportion of patients with the same first and second $b / t s D M A R D s$ may be explained by a misclassified stop (e.g., drug holidays), but it may as well be indicative of patients restarting a therapy they previously did well on. Further research on the patterns of stopping $b /$ tsDMARDs would be of interest.

\section{Conclusions}

In conclusion, this study provided a clinical picture of PsA and RA patients in Switzerland across different BMI categories. Obesity prevalence was higher in PsA and RA compared to the general Swiss population, and PsA and RA patients with obesity had worse disease activity and lower QoL in comparison with the corresponding normal weight groups. In the PsA cohort, the findings on disease activity (DAPSA) in obese patients were mainly driven by CRP levels and the patient assessment on disease activity and pain, but the results remained consistent when excluding CRP levels from the equation (cDAPSA). In the RA cohort, the results on disease activity (DAS28) in obese patients were primarily attributable to TJC and ESR or CRP levels. Finally, these findings suggest that BMI should be considered when treating or studying PsA and RA patients.

Supplementary Materials: The following are available online at https:/ /www.mdpi.com/article/10 .3390/jcm10143194/s1: Supplementary Equations (S1)-(S6); Supplementary Figure S1. Flow chart reflecting the patient inclusion process; Supplementary Table S1. Characteristics of psoriatic arthritis (PsA) patients starting their first biologic or targeted synthetic disease-modifying anti-rheumatic drug (b/tsDMARD) treatment. Additional variables to complement Tables 2 and 3; Supplementary Table S2. Characteristics of rheumatoid arthritis (RA) patients starting their first biologic or targeted synthetic disease-modifying anti-rheumatic drug (b/tsDMARD) treatment. Additional variables to complement Tables 5 and 6; Supplementary Table S3. First and second biologic or targeted synthetic disease-modifying anti-rheumatic drug (b/tsDMARD) treatment among the psoriatic arthritis (PsA) patients. Only those PsA patients with recorded information on both treatments were included; Supplementary Table S4. First and second biologic or targeted synthetic disease-modifying antirheumatic drug (b/tsDMARD) treatment among the rheumatoid arthritis (RA) patients. Only those RA patients with recorded information on both treatments were included.

Author Contributions: Conceptualization, E.V.-Y., T.B., and A.M.B.; methodology, E.V.-Y.; formal analysis, E.V.-Y.; investigation, E.V.-Y.; resources, A.M.B.; data curation, E.V.-Y.; writing—original draft preparation, E.V.-Y.; writing-review and editing, E.V.-Y., T.B., B.M., A.F., and A.M.B.; visualization, E.V.-Y.; supervision, A.M.B.; project administration E.V.-Y. and A.M.B. All authors have read and agreed to the published version of the manuscript.

Funding: This research received no external funding.

Institutional Review Board Statement: The study was reviewed by the ethics commission of the Canton of Zurich (KEK: Req-2020-00045). The researchers received pseudonymized data without access to the code key; therefore, a full ethics authorization was waived by the commission.

Informed Consent Statement: Patients included in the SCQM registry provide signed informed consent prior to enrolment in accordance with the Declaration of Helsinki and withdrawal of participation was possible at any time. 
Data Availability Statement: Restrictions apply to the availability of these data. Data were obtained from the Swiss Clinical Quality Management in Rheumatic Diseases (SCQM) and are available after having received approval and permission from the license holder (SCQM).

Acknowledgments: We thank all patients and rheumatologists contributing to the SCQM registry, as well as the entire SCQM staff. A list of rheumatology offices and hospitals that contribute to the SCQM registry can be found at http:/ / www.scqm.ch/institutions (accessed on 12 May 2021). A list of financial supporters of SCQM can be found at http:/ / www.scqm.ch/sponsors (accessed on 12 May 2021). AMB acknowledges that her professorship is partly endowed by the Swiss National Pharmacy Association (PharmaSuisse) and the ETH Foundation.

Conflicts of Interest: The authors declare no conflict of interest related to this work.

\section{References}

1. Kopelman, P.G. Obesity as a Medical Problem. Nature 2000, 404, 635-643. [CrossRef] [PubMed]

2. Body Mass Index (BMI) by Sex, Age and Educational Attainment Level (Last Update: 08-02-2021). Available online: https: / / appsso.eurostat.ec.europa.eu/nui/show.do?dataset=hlth_ehis_bm1e\&lang=en (accessed on 14 May 2021).

3. Statistik, B. für Übergewicht und Adipositas—Schweizerische Gesundheitsbefragung 2017-Korrigierte Version 25 September 2020 I Publikation. Available online: https:/ / www.bfs.admin.ch/bfs/de/home/statistiken/gesundheit/erhebungen/sgb. assetdetail.14147705.html (accessed on 21 January 2021).

4. Versini, M.; Jeandel, P.-Y.; Rosenthal, E.; Shoenfeld, Y. Obesity in Autoimmune Diseases: Not a Passive Bystander. Autoimmun. Rev. 2014, 13, 981-1000. [CrossRef]

5. Gremese, E.; Tolusso, B.; Gigante, M.R.; Ferraccioli, G. Obesity as a Risk and Severity Factor in Rheumatic Diseases (Autoimmune Chronic Inflammatory Diseases). Front Immunol. 2014, 5. [CrossRef] [PubMed]

6. Iannone, F.; Lopalco, G.; Rigante, D.; Orlando, I.; Cantarini, L.; Lapadula, G. Impact of Obesity on the Clinical Outcome of Rheumatologic Patients in Biotherapy. Autoimmun. Rev. 2016, 15, 447-450. [CrossRef] [PubMed]

7. Finckh, A.; Turesson, C. The Impact of Obesity on the Development and Progression of Rheumatoid Arthritis. Ann. Rheum. Dis. 2014, 73, 1911-1913. [CrossRef]

8. George, M.D.; Baker, J.F. The Obesity Epidemic and Consequences for Rheumatoid Arthritis Care. Curr. Rheumatol. Rep. 2016, 18, 6. [CrossRef]

9. Daïen, C.I.; Sellam, J. Obesity and Inflammatory Arthritis: Impact on Occurrence, Disease Characteristics and Therapeutic Response. RMD Open 2015, 1, e000012. [CrossRef]

10. Albrecht, K.; Richter, A.; Callhoff, J.; Huscher, D.; Schett, G.; Strangfeld, A.; Zink, A. Body Mass Index Distribution in Rheumatoid Arthritis: A Collaborative Analysis from Three Large German Rheumatoid Arthritis Databases. Arthritis Res. 2016, 18. [CrossRef]

11. Bhole, V.M.; Choi, H.K.; Burns, L.C.; Vera Kellet, C.; Lacaille, D.V.; Gladman, D.D.; Dutz, J.P. Differences in Body Mass Index among Individuals with PsA, Psoriasis, RA and the General Population. Rheumatology 2012, 51, 552-556. [CrossRef]

12. Eder, L.; Thavaneswaran, A.; Chandran, V.; Cook, R.J.; Gladman, D.D. Obesity Is Associated with a Lower Probability of Achieving Sustained Minimal Disease Activity State among Patients with Psoriatic Arthritis. Ann. Rheum. Dis. 2015, 74, 813-817. [CrossRef]

13. Eder, L.; Abji, F.; Rosen, C.F.; Chandran, V.; Gladman, D.D. The Association Between Obesity and Clinical Features of Psoriatic Arthritis: A Case-Control Study. J. Rheumatol. 2017, 44, 437-443. [CrossRef] [PubMed]

14. Labitigan, M.; Bahče-Altuntas, A.; Kremer, J.M.; Reed, G.; Greenberg, J.D.; Jordan, N.; Putterman, C.; Broder, A. Higher Rates and Clustering of Abnormal Lipids, Obesity, and Diabetes Mellitus in Psoriatic Arthritis Compared with Rheumatoid Arthritis. Arthritis Care Res. 2014, 66, 600-607. [CrossRef]

15. Højgaard, P.; Glintborg, B.; Kristensen, L.E.; Gudbjornsson, B.; Love, T.J.; Dreyer, L. The Influence of Obesity on Response to Tumour Necrosis Factor- $\alpha$ Inhibitors in Psoriatic Arthritis: Results from the DANBIO and ICEBIO Registries. Rheumatology 2016, 55, 2191-2199. [CrossRef] [PubMed]

16. Liu, Y.; Hazlewood, G.S.; Kaplan, G.G.; Eksteen, B.; Barnabe, C. Impact of Obesity on Remission and Disease Activity in Rheumatoid Arthritis: A Systematic Review and Meta-Analysis. Arthritis Care Res. 2017, 69, 157-165. [CrossRef]

17. Di Minno, M.N.D.; Peluso, R.; Iervolino, S.; Lupoli, R.; Russolillo, A.; Scarpa, R.; di Minno, G. Obesity and the Prediction of Minimal Disease Activity: A Prospective Study in Psoriatic Arthritis. Arthritis Care Res. 2013, 65, 141-147. [CrossRef]

18. Singh, S.; Facciorusso, A.; Singh, A.G.; Vande Casteele, N.; Zarrinpar, A.; Prokop, L.J.; Grunvald, E.L.; Curtis, J.R.; Sandborn, W.J. Obesity and Response to Anti-Tumor Necrosis Factor- $\alpha$ Agents in Patients with Select Immune-Mediated Inflammatory Diseases: A Systematic Review and Meta-Analysis. PLOS ONE 2018, 13, e0195123. [CrossRef] [PubMed]

19. Sandberg, M.E.C.; Bengtsson, C.; Källberg, H.; Wesley, A.; Klareskog, L.; Alfredsson, L.; Saevarsdottir, S. Overweight Decreases the Chance of Achieving Good Response and Low Disease Activity in Early Rheumatoid Arthritis. Ann. Rheum. Dis. 2014, 73, 2029-2033. [CrossRef] [PubMed]

20. Lupoli, R.; Pizzicato, P.; Scalera, A.; Ambrosino, P.; Amato, M.; Peluso, R.; Di Minno, M.N.D. Impact of Body Weight on the Achievement of Minimal Disease Activity in Patients with Rheumatic Diseases: A Systematic Review and Meta-Analysis. Arthritis. Res. 2016, 18, 297. [CrossRef] [PubMed] 
21. Moroni, L.; Farina, N.; Dagna, L. Obesity and Its Role in the Management of Rheumatoid and Psoriatic Arthritis. Clin. Rheumatol. 2020, 39, 1039-1047. [CrossRef]

22. Weijers, J.M.; Müskens, W.D.; van Riel, P.L.C.M. Effect of Significant Weight Loss on Disease Activity: Reason to Implement This Non-Pharmaceutical Intervention in Daily Clinical Practice. RMD Open 2021, 7. [CrossRef] [PubMed]

23. Minno, M.N.D.D.; Peluso, R.; Iervolino, S.; Russolillo, A.; Lupoli, R.; Scarpa, R.; on behalf of the CaRRDs Study Group. Weight Loss and Achievement of Minimal Disease Activity in Patients with Psoriatic Arthritis Starting Treatment with Tumour Necrosis Factor $\alpha$ Blockers. Ann. Rheum. Dis. 2014, 73, 1157-1162. [CrossRef] [PubMed]

24. Stavropoulos-Kalinoglou, A.; Metsios, G.S.; Panoulas, V.F.; Nevill, A.M.; Jamurtas, A.Z.; Koutedakis, Y.; Kitas, G.D. Underweight and Obese States Both Associate with Worse Disease Activity and Physical Function in Patients with Established Rheumatoid Arthritis. Clin. Rheumatol. 2009, 28, 439-444. [CrossRef] [PubMed]

25. Die SCQM Foundation (Swiss Clinical Quality Management in Rheumatic Diseases). Available online: https:/ /www.scqm.ch/ (accessed on 12 May 2021).

26. World Health Organization (WHO), Body Mass Index (BMI). Available online: https://www.euro.who.int/en/health-topics/ disease-prevention/nutrition/a-healthy-lifestyle/body-mass-index-bmi (accessed on 14 May 2021).

27. Gonçalves, R.S.G.; de Almeida Martins, L.M.; de Ataide Mariz, H.; Dantas, A.T.; Duarte, A.L.B.P. DAPSA versus CDAPSA: Do We Need to Use CRP ? Ann. Rheum. Dis. 2019. [CrossRef]

28. R Core Team. R: A Language and Environmental for Statistical Computing; R Foundation for Statistical Computing: Vienna, Austria, 2018; Available online: https: / / www.r-project.org/ (accessed on 14 May 2021).

29. GraphPad Prism Version 9.0.2 for Windows. GraphPad Software: San Diego, CA, USA. Available online: https://www.graphpad. $\mathrm{com} /$ (accessed on 14 May 2021).

30. State of Health in the EU Denmark Country Health Profile. 2019. Available online: https://ec.europa.eu/health/sites/default/ files/state/docs/2019_chp_da_english.pdf (accessed on 10 May 2021).

31. Heilsa Og Líðan I'slendinga. 2012. Available online: https://www.landlaeknir.is/servlet/file/store93/item22830 /Framkvaemdaskyrsla_2012_loka.pdf (accessed on 10 May 2021).

32. Klaasen, R.; Wijbrandts, C.A.; Gerlag, D.M.; Tak, P.P. Body Mass Index and Clinical Response to Infliximab in Rheumatoid Arthritis. Arthritis Rheum. 2011, 63, 359-364. [CrossRef] [PubMed]

33. Escalante, A.; Haas, R.W.; del Rincón, I. Paradoxical Effect of Body Mass Index on Survival in Rheumatoid Arthritis: Role of Comorbidity and Systemic Inflammation. Arch. Intern. Med. 2005, 165, 1624-1629. [CrossRef]

34. Vidal, C.; Barnetche, T.; Morel, J.; Combe, B.; Daïen, C. Association of Body Mass Index Categories with Disease Activity and Radiographic Joint Damage in Rheumatoid Arthritis: A Systematic Review and Metaanalysis. J. Rheumatol. 2015, 42, 2261-2269. [CrossRef]

35. Gota, C.E.; Kaouk, S.; Wilke, W.S. Fibromyalgia and Obesity: The Association Between Body Mass Index and Disability, Depression, History of Abuse, Medications, and Comorbidities. J. Clin. Rheumatol. 2015, 21, 289-295. [CrossRef]

36. Arranz, L.-I.; Rafecas, M.; Alegre, C. Effects of Obesity on Function and Quality of Life in Chronic Pain Conditions. Curr. Rheumatol. Rep. 2013, 16, 390. [CrossRef] [PubMed]

37. Heo, M.; Allison, D.B.; Faith, M.S.; Zhu, S.; Fontaine, K.R. Obesity and Quality of Life: Mediating Effects of Pain and Comorbidities. Obes. Res. 2003, 11, 209-216. [CrossRef]

38. Armstrong, D.J.; McCausland, E.M.; Quinn, A.D.; Wright, G.D. Obesity and Cardiovascular Risk Factors in Rheumatoid Arthritis. Rheumatology 2006, 45, 782, author reply 782-783. [CrossRef]

39. Al-Goblan, A.S.; Al-Alfi, M.A.; Khan, M.Z. Mechanism Linking Diabetes Mellitus and Obesity. Diabetes Metab. Syndr. Obes. 2014, 7, 587-591. [CrossRef]

40. Thomsen, R.S.; Nilsen, T.I.L.; Haugeberg, G.; Gulati, A.M.; Kavanaugh, A.; Hoff, M. Adiposity and Physical Activity as Risk Factors for Developing Psoriatic Arthritis: Longitudinal Data From a Population-Based Study in Norway. Arthritis Care Res. 2021, 73, 432-441. [CrossRef]

41. Feng, X.; Xu, X.; Shi, Y.; Liu, X.; Liu, H.; Hou, H.; Ji, L.; Li, Y.; Wang, W.; Wang, Y.; et al. Body Mass Index and the Risk of Rheumatoid Arthritis: An Updated Dose-Response Meta-Analysis. Biomed. Res. Int. 2019, 2019. [CrossRef]

42. Perez-Chada, L.M.; Merola, J.F. Comorbidities Associated with Psoriatic Arthritis: Review and Update. Clin. Immunol. 2020, 214, 108397. [CrossRef] [PubMed]

43. El Miedany, Y. Comorbidity in Rheumatic Diseases; Springer Science + Business Media: New York, NY, USA, 2017; ISBN 978-3-31959962-5.

44. D'Agostino, M.-A.; Alten, R.; Mysler, E.; Le Bars, M.; Ye, J.; Murthy, B.; Heitzmann, J.; Vadanici, R.; Ferraccioli, G. Body Mass Index and Clinical Response to Intravenous or Subcutaneous Abatacept in Patients with Rheumatoid Arthritis. Clin. Rheumatol. 2017, 36, 2655-2665. [CrossRef] [PubMed]

45. Gardette, A.; Ottaviani, S.; Sellam, J.; Berenbaum, F.; Lioté, F.; Fautrel, B.; Palazzo, E.; Meyer, A.; Sibilia, J.; Dieudé, P. Body Mass Index and Response to Abatacept in Rheumatoid Arthritis. Eur. J. Clin. Invest. 2016, 46, 1048-1052. [CrossRef]

46. Iannone, F.; Courvoisier, D.S.; Gottenberg, J.E.; Hernandez, M.V.; Lie, E.; Canhão, H.; Pavelka, K.; Hetland, M.L.; Turesson, C.; Mariette, X.; et al. Body Mass Does Not Impact the Clinical Response to Intravenous Abatacept in Patients with Rheumatoid Arthritis. Analysis from the "pan-European Registry Collaboration for Abatacept (PANABA). Clin. Rheumatol. 2017, 36, 773-779. [CrossRef] 
47. Mariette, X.; Alten, R.; Nüßlein, H.G.; Galeazzi, M.; Lorenz, H.-M.; Cantagrel, A.; Chartier, M.; Poncet, C.; Rauch, C.; Le Bars, M. The Effect of Body Mass Index on Clinical Response to Abatacept as a First-Line Biologic for Rheumatoid Arthritis: 6-Month Results from the 2-Year, Observational, Prospective ACTION Study. Jt. Bone Spine 2016, 84, 571-576. [CrossRef]

48. Ottaviani, S.; Gardette, A.; Roy, C.; Tubach, F.; Gill, G.; Palazzo, E.; Meyer, O.; Dieudé, P. Body Mass Index and Response to Rituximab in Rheumatoid Arthritis. Jt. Bone Spine 2015, 82, 432-436. [CrossRef]

49. Gardette, A.; Ottaviani, S.; Sellam, J.; Berenbaum, F.; Lioté, F.; Meyer, A.; Sibilia, J.; Fautrel, B.; Palazzo, E.; Dieudé, P. Body Mass Index and Response to Tocilizumab in Rheumatoid Arthritis: A Real Life Study. Clin. Rheumatol. 2016, 35, 857-861. [CrossRef] [PubMed]

50. Pers, Y.-M.; Godfrin-Valnet, M.; Lambert, J.; Fortunet, C.; Constant, E.; Mura, T.; Pallot-Prades, B.; Jorgensen, C.; Maillefert, J.-F.; Marotte, H.; et al. Response to Tocilizumab in Rheumatoid Arthritis Is Not Influenced by the Body Mass Index of the Patient. J. Rheumatol. 2015, 42, 580-584. [CrossRef] [PubMed]

51. Overweight and Obesity-BMI Statistics—Statistics Explained. Available online: https://ec.europa.eu/eurostat/statisticsexplained/index.php/Overweight_and_obesity_-_BMI_statistics (accessed on 23 February 2021).

52. Stavropoulos-Kalinoglou, A.; Metsios, G.S.; Koutedakis, Y.; Nevill, A.M.; Douglas, K.M.; Jamurtas, A.; van Zanten, J.J.C.S.V.; Labib, M.; Kitas, G.D. Redefining Overweight and Obesity in Rheumatoid Arthritis Patients. Ann. Rheum. Dis. 2007, 66, 1316-1321. [CrossRef]

53. Summers, G.D.; Metsios, G.S.; Stavropoulos-Kalinoglou, A.; Kitas, G.D. Rheumatoid Cachexia and Cardiovascular Disease. Nat Rev. Rheumatol. 2010, 6, 445-451. [CrossRef]

54. Kamalaraj, N.; El-Haddad, C.; Hay, P.; Pile, K. Systematic Review of Depression and Anxiety in Psoriatic Arthritis. Int. J. Rheum. Dis. 2019, 22, 967-973. [CrossRef]

55. McDonough, E.; Ayearst, R.; Eder, L.; Chandran, V.; Rosen, C.F.; Thavaneswaran, A.; Gladman, D.D. Depression and Anxiety in Psoriatic Disease: Prevalence and Associated Factors. J. Rheumatol. 2014, 41, 887-896. [CrossRef] [PubMed]

56. Husni, M.E. Comorbidities in Psoriatic Arthritis. Rheum. Dis. Clin. N. Am. 2015, 41, 677-698. [CrossRef]

57. Matcham, F.; Rayner, L.; Steer, S.; Hotopf, M. The Prevalence of Depression in Rheumatoid Arthritis: A Systematic Review and Meta-Analysis. Rheumatology 2013, 52, 2136-2148. [CrossRef] 